\title{
A Decision Support System for Moving Workloads to Public Clouds
}

\author{
Mohammad Firoj Mithani \\ Unisys Global Services India \\ mohammad.mithani@unisys.com
}

\author{
Michael A. Salsburg, Ph.D. \\ Unisys Corporation, USA \\ michael.salsburg@unisys.com
}

\author{
Shrisha Rao, Ph.D. \\ IIIT-Bangalore, India \\ shrao@ieee.org
}

\begin{abstract}
The current economic environment is compelling CxOs to look for better IT resource utilization in order to get more value from their IT investments and reuse existing infrastructure to support growing business demands. How to get more from less? How to reuse the resources? How to minimize the Total Cost of Ownership (TCO) of underlying IT infrastructure and data center operation cost? How to improve Return On Investment (ROI) to remain profitable and transform the IT cost center into a profit center? All of these questions are now being considered in light of emerging 'Public Cloud Computing' services. Cloud Computing is a model for enabling resource allocation to dynamic business workloads in a real time manner from a pool of free resources in a cost effective manner. Providing resource on demand at cost effective pricing is not the only criteria when determining if a business service workload can be moved to a public cloud. So what else must $\mathrm{CxOs}$ consider before they migrate to public cloud environments? There is a need to validate the business applications and workloads in terms of technical portability and business requirements/compliance so that they can be deployed into a public cloud without considerable customization. This validation is not a simple task.
\end{abstract}

In this paper, we will discuss an approach and the analytic tooling which will help CxOs and their teams to automate the process of identifying business workloads that should move to a public cloud environment, as well as understanding its cost benefits. Using this approach, an organization can identify the most suitable business service workloads which could be moved to a public cloud environment from a private data center without re-architecting the applications or changing their business logic. This approach helps automate the classification and categorization of workloads into various categories. For example, Business Critical (BC) and Non-business Critical (NBC) workloads can be identified based on the role of business services within the overall business function. The approach helps in the assessment of public cloud providers on the basis of features and constraints. This approach provides consideration for industry compliance and the price model for hosting workloads on a pay-per-use basis. Finally, the inbuilt analytics in the tool find the 'best-fit' cloud provider for hosting the business service workload. 'Bestfit' is based on analysis and outcomes of the previously mentioned steps.

Today, the industry follows a manual time consuming process for workload identification, workload classification and cloud provider assessment to find the best-fit for business service workload hosting. The suggested automated approach enables an organization to reduce cost and time when deciding to move to a public cloud environment. The proposed automated approach accelerates the entire process of leveraging cloud benefits, through an effective, informed, fact-based decision process.

\section{INTRODUCTION}

Cloud Computing provides a computing model that can transform IT Support and Services (ISS) from a cost centre to a profit centre by optimizing existing IT infrastructure resource utilization. This can be accomplished through effective resource allocation \& de-allocation on demand and in a real-time manner. On-Demand, utility computing reduces capital expenses (CapEx) while automation reduces operating expenses (OpEx) to respond proactively to unpredictable business service demands for IT resources through allocation of resources on need basis. In addition, improving server resource utilization through virtualization helps organizations to keep their IT spending as low as possible. Two of the top priorities for CIOs' in 2010 include adapting virtualization and using a cloud computing approach to improve overall IT infrastructure investment to support business growth [1]. There are three choices to keep the IT spending under control without affecting the business growth: moving workloads to a public cloud environment, building a Private Cloud, or by adopting a Hybrid Cloud approach that uses both public and private clouds [2].

On the basis of Net Present Value (NPV), Benefit-Cost Ratio (BCR) and Discounted Payback Period (DPP), moving business services to public clouds can be the most cost effective choice [4]. The overall benefits of moving to cloud environments are depicted in Figure 1 [3]. Cloud computing really comes into focus only when you think about what IT always needs: a way to increase capacity or add capabilities on the fly without investing in new IT infrastructure/hardware, training personnel, or new data center facilities. Cloud computing encompasses any subscription-based or pay-peruse service that can be utilized in real time over the Internet, thus extending IT's existing capabilities [5].

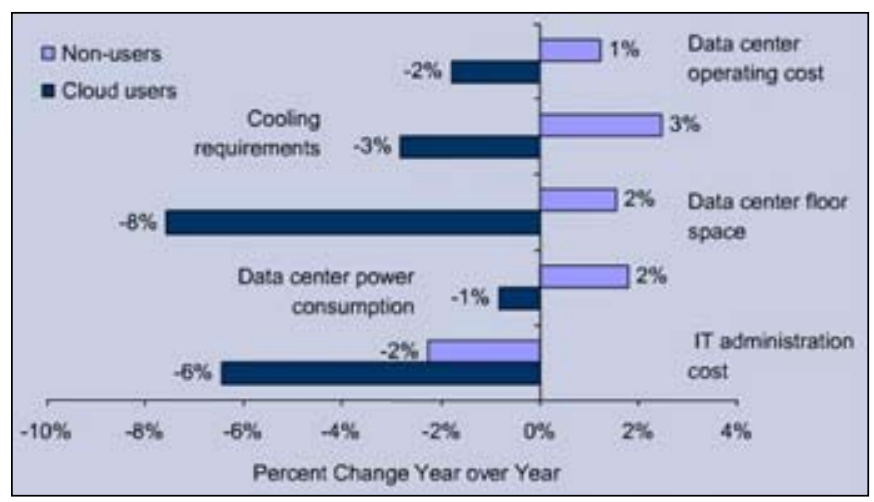

Fig. 1. Cloud User IT Cost Savings 
Organizations are eager to move business workloads from private data centers to public cloud to reap the cloud computing benefits. The analytics and the tooling, explained below, assist the organization to capture business workload attributes through creating workload profiles. The workload profile is comprised of a set of business services. Each business service defines a specific service. These business services, in turn, define the necessary business applications, data, technical and business characteristics for that service. A workload is the collection of all of these attributes. The tool discussed in this paper uses analytics that assign weights to individual attributes on the basis of nature of the workload. Similarly, the tool maintains the database of public cloud environment attributes in the form of individual cloud profiles. Each profile consists of a cloud provider's features, the environmental constraints and other specific details. The analytics find the most suitable public cloud provider to host the business workload to meet or exceed the objectives of moving a workload to the public cloud. The tool reduces time and effort required to define the workload, as well as matching the workload attributes to the cloud environment attributes.

\section{HOW IS THIS BEING DONE TODAY?}

Before any organization moves the business workload to a public cloud, the movement has to be justified in terms of benefits to business as well as technology support from the cloud provider. There is no disagreement that every business workload has some business logic to execute business services, and every such business service is made up of software hosted on hardware. So, if an organization is keen on moving the workload to public cloud, then it has to find out if the existing workload can be deployed in public cloud environment without considerable modifications to the way the business works. Similarly, the organization has to ensure that the public cloud environment meets all necessary hardware and software pre-requisites to host the workload without affecting the performance of the business services. To ensure the satisfaction of business as well as technology requirements, the business and technology organizations need to understand and document all the pre-requisites to host the business workload. Considering the dynamics of the public cloud environment, the organization need experts to understand and analyze the public cloud provider's offerings, price model, and industry compliance. However, there are very few Infrastructure as a Service (IaaS) [7] providers in the market. Each IaaS provider has many business and implementation partners to facilitate the client's workload building and movement to the cloud. The public cloud offerings are very complex, which makes it difficult to understand the best strategy for movement to a specific cloud in terms of technologies, and terminologies. Cloud analysis is a specialized domain of expertise, where only the public cloud providers' business partners, implementation advisors can assist clients/organizations to find the best-fit for a public cloud, given their particular business workload. These implementation advisors need enough time to understand the business logic and technology dependencies of the business workloads. Hence, the organizations need to invest a lot of time in educating the advisors to understand the business process. The activity takes a lot of time and effort to enable the business leaders of the organization to make fact based decisions on workload movement to public cloud.

This is where the Cloud Decision Support System (CDSS) can be used to automate many of these manual processes for workload analysis and public cloud assessment to identify the most suitable cloud environment to host the workload. This paper presents a formal process for workload assessment and migration to cloud.

\section{THE CLOUD DECISION SUPPORT SYSTEM}

The Cloud Decision Support System (CDSS) is easy to use for any organization to build a repository of the business service workload profiles. The profile can be updated as the workload attributes change. The system provides an easy to use interface to create the public cloud providers' profiles and then populate the profiles with the cloud offerings' features. The system is being implemented as a 2-tier web application with a web-based user interface which can be accessed on the Internet, thus providing the ubiquity of cloud computing. A back end data tier is implemented as a service where the web server is located. The CDSS database maintains the necessary attributes of the business service workload and the public cloud environments. The system is quick to implement and easy to use for any organization. Let us have a look inside the system.

\subsection{Public Cloud Discovery and Analysis}

The system provides a systematic process for creating profiles of cloud providers to capture the necessary attributes. It can be achieved manually or automated using simple scripts. Every public cloud provider is assigned with a unique Public Cloud Identifier (PCID) which is mapped to the cloud provider's name. The cloud profile helps in capturing all the features and offerings of the provider.

Building the cloud provider's profile helps in maintaining the entire feature details as well as information regarding compliance and certifications achieved by the provider for the cloud environment. A cloud provider can be classified into various categories viz., Financial Cloud, Educational Cloud, Social Network Cloud, Marketing Cloud, Sales and Distribution Cloud, etc. The classification of any public cloud into any of these categories depends on certain criteria. For instance, a Financial Cloud must have achieved the Statement on Auditing Standards (SAS) 70 audit reports, a Data Protection and Information Security (ISO 27001) certification. Other statements may be required from the Federal Deposit Insurance Corporation (FDIC), Federal Financial Institutions Examination Council (FFIEC), Office of the Comptroller of the Currency (OCC), and National Institute of Standards and Technology (NIST) and perhaps others, depending on the company's governance policies. So far, only Unisys Secure 
Cloud has been audited and recommended by independent bodies for these certifications [13]. The classification of the public cloud provider helps in quickly eliminating other non-compliant cloud providers for specific types of workloads. CDSS identifies and classifies every public cloud provider after analyzing the entire profile and each attribute of the cloud provider. Whenever there is a change in any of the attributes of the cloud, the system re-processes the data to analyze and re-classify the public cloud environment. Figure 2 shows a graphical representation of the fact gathering process. Frequent analysis of the cloud providers' profile helps in finding the bestfit cloud environment for the target workloads.

Have a look at the Table 1; it depicts the ranking of five sample public cloud providers regarding their applicability for financial business workloads. In the table, the Public Cloud2 (PC2) is the most preferred public cloud environment from the available providers on the basis of its attributes to host financial workloads.

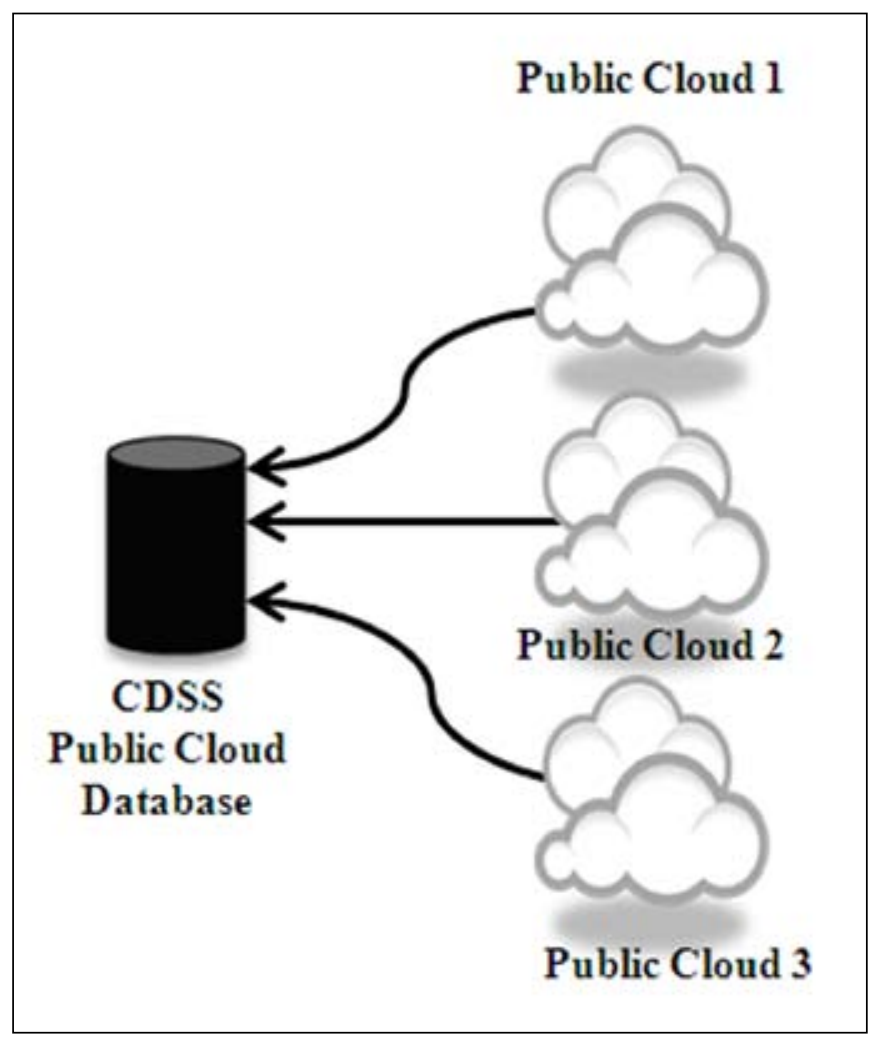

Fig. 2. Public Cloud Discovery and Analysis

Table 1. Cloud Provider Ranking - Business

\begin{tabular}{|c|c|c|c|c|}
\hline CP & $\begin{array}{c}\text { SAS 70 } \\
\text { Type I }\end{array}$ & $\begin{array}{c}\text { SAS 70 } \\
\text { Type II }\end{array}$ & $\begin{array}{c}\text { ISO /IEC } \\
27001\end{array}$ & CPR \\
\hline PC1 & Yes & No & No & 4 \\
\hline PC2 & Yes & Yes & Yes & 1 \\
\hline PC3 & Yes & Yes & No & 2 \\
\hline PC4 & Yes & No & Yes & 3 \\
\hline PC5 & No & No & No & 5 \\
\hline
\end{tabular}

The entire ranking is based on SAS 70 Type I and Type II as well as ISO/IEC 27001 compliance status. CDSS considers such multiple attributes to classify and rank the public cloud provider. Our Cloud Provider Ranking (CPR) approach precisely helps to identify and classify any cloud provider to host specific workloads. As you add more attributes, the complexity of the rank calculation increases combinatorially. The most preferred rank can be identified by the organization to decide on which public cloud environment the workload should be hosted.

Another example could be the workload of a health service provider organization. For such a workload, Health Insurance Portability and Accountability Act (HIPPA) is more valuable than SAS 70 Type I or Type II. In such cases, the organization is free to set the higher weighing for HIPPA in relationship to a SAS 70 attribute. Organization can customize the public cloud analysis approach through customizing the CDSS analytics as per the nature of the business workload. Along with the industry specific compliance, other attributes which are equally important to rank any cloud environment are IT infrastructure Availability, Disaster Recovery capability, Service Level Agreement (SLA) and Business Service Level Objectives (SLO). In addition, there should be considerations for the level of technical support provided by the cloud provider, clearly defined functional as well as the hierarchical escalation matrix, physical security of the hosted servers/data center, price models, terms of exit, efforts of moving to public cloud environment, use of existing licenses, IT support framework such as ITIL (IT Infrastructure Library) and data center certification level from Tier I to Tier IV [6].

In general, cloud providers do not facilitate the actual workload identification, customization, migration, deployment and ongoing support. These activities are mostly provided by business partners and implementation advisors. For example, Amazon Web Service (AWS) has business partners like RightScale, CloudSwitch and enStratus to facilitate the actual workload building, configuration, database synchronization, standard image customization, workload management and monitoring services. The system presented here considers the availability of the business partner of the target cloud provider, their past experiences, number of existing clients, total number of successful migrations and cost of services. Organizations can weigh the analysis for each of these attributes to find the optimal business partner for the cloud provider under consideration.

The attributes discussed so far is industry specific to any particular business workload and is related to Service Delivery. The other group 
of attributes the organization must consider before actual execution of workload migration to cloud environment is the technology. Very few Infrastructure as a Service (IaaS) providers provide the physical as well as virtual servers for workload hosting in the manner of the Unisys Secure Cloud (USC). Most IaaS providers provide only virtual machines and virtual resources to host the business workloads. One example is AWS Elastic Compute Cloud (EC2). If the business workload is already virtualized using any of the virtualization technologies such as VMware, Xen, KVM, then it could be easily hosted with any of the IaaS providers. But some workloads cannot be hosted on virtual servers, such as legacy workloads that are not qualified to execute in a VM. Another example is a workload that requires very precise service level objectives. They may not run as predicted if they are running in a VM that is sharing a physical host with other VMs. If the organization prefers to host the application on a dedicated physical server then there are limited choices. Overall, it is preferred to have business workloads in virtual image formats to provide maximum mobility regarding public cloud environments. Having said that, virtual formats vary and the customer's format may not be supported by the target IaaS provider. For example, Unisys Secure Cloud (USC) currently supports the VMware hypervisor, while AWS EC2 supports a customized Xen hypervisor, thus limiting Virtual Machine mobility from one cloud to another. In such a case, if the organization has currently implemented their workloads in VMware format, and wants to move to AWS EC2, a virtual to virtual (V2V) image conversion is required. Post conversion, the organization needs to test the performance and behavior of the application in its new virtualization format.

There are actually two steps in workload movement from a private data center to a public cloud. First is the conversion from source to target virtualization formats as discussed above. Once the conversion is completed, then the workload must be transported from the private data center into the public cloud. The Distributed Management Task Force (DMTF) has released a virtualization packaging and distribution standard called Open Virtualization Format (OVF). OVF provides options to convert single VM or multitier business service workloads in multiple target virtual machine formats for workload distribution. OVF provides data compression, data integrity and encryption feature for efficient and secure workload distribution. But OVF does not guarantee universal portability or install-ability of VMs across all of the hypervisors [8]. The limitation is not really caused by the OVF format. OVF supports all the latest virtual disk formats. The limitation is caused by unsupported virtual disk formats by the target cloud's virtualization hypervisor.

Other factors which are considered by CDSS while identifying and classifying cloud environments are supported Operating Systems (OS), Data Base Management Systems (DBMS), and Application development environments. On the hardware side, underlying server, storage, network, and load balancer hardware must be considered along with the dynamic scale-in, scale-out, scale-up and scale-down capabilities. Data protection in motion (DIM) and data protection at rest (DAR) in multitenant shared environments may be a concern. Additional capabilities could include resource demand forecasting for the business applications, dynamic business service discovery, end to end business service transaction monitoring, alerting, event logging, auto-incident generation, self service console etc. The organizations can set the weights for these attributes and features (or the lack of the features).

Have a look at the Table 2, where we list attributes for three commercial public cloud providers (CP). Four attributes and their impact on the Cloud Provider Rating (CPR) are shown in the table.

From the ranking, it shows that USC is the most preferred public cloud environment for any VMware based virtualized or physical server based workloads which require a high level of data protection for the client's business data in motion.

Table 2. Cloud Provider Ranking - Technology

\begin{tabular}{|c|c|c|c|c|c|}
\hline CPs & $\begin{array}{c}\text { Provide } \\
\text { Physical } \\
\text { Servers }\end{array}$ & $\begin{array}{c}\text { Provide } \\
\text { Virtual } \\
\text { Servers }\end{array}$ & $\begin{array}{c}\text { Virtual } \\
\text { Machine } \\
\text { format }\end{array}$ & DIM & C \\
P \\
R
\end{tabular}

\subsection{Workload Identification and Analysis}

The application workloads and their constituent business service identification, profiling and analysis are as important as the public cloud assessment process through cloud discovery and profiling [10]. The identification of business services and application workloads to move to the public cloud environment is internal to the business and should be based on tangible objectives regarding movement to the cloud. What is the motivation? It can be the reduction of direct or indirect costs for hosting and managing the workloads. On the other hand, there may be a requirement to engage the most efficient service provider to improve the IT infrastructure ROI supporting the core business applications or both of these motivations may be considered 
simultaneously. After identifying the business applications, CDSS helps in capturing the business as well as technical attributes of the workload through workload profiling. This step is important to understand the nature of workload in the context of business as well as required underlying technology dependencies.

Traditionally, the workloads that are moved to the public cloud environment are -

- Workloads that require extreme elasticity, such as 3 servers today, 1,000 tomorrow and 2 servers the next day

- Test and pre-production systems

- Mature, contextual applications such as email and collaboration applications that are not considered part of the business' core technology focus

- Software development environments

- Batch processing jobs with limited security requirements

- Isolated workloads where latency between components is not an issue

- Storage solutions/storage as a service

- Backup solutions/backup and restore as a service

- Data intensive workloads if the provider has accompanying storage as a service [12].

Let us take an example of a typical financial business service workload. The application can require a single server or $\mathrm{n}$-tier implementation. It could require a Real-time Online Transaction Processing(OLTP)environment. Suchapplications require constant access to storage, has hundreds or thousands of mission-critical, confidential business transactions arriving through its Web Tier, traversing to an Application Tier and finally retrieving and committing data/information from/to Database Tier, with a requirement to be available around the clock. In this case, the workload has an attribute indicating its service level in terms of availability. High availability is required for the entire IT infrastructure supporting all the tiers, including but not limited to underlying networks infrastructure, storage devices, hosting server hardware, implemented virtualization applications, as well as uninterrupted data exchange with external business applications. Such workloads may require strict Access Control Lists (ACLs) to be maintained. The hosting facility (data center) must be audited for SAS 70 Type II, FDIC, FFIEC, OCC, NIST, Sarbanes-Oxley and Gramm-Leach-Bliley Act, (Financial Services Modernization Act of 1999) (GLBA). Entry in the data center as well as raised-floors must be secured by multi-factor access control system. Disaster Recovery (DR) and Business Continuity Planning (BCP) for the application workloads must meet business governance policies at server level, at entire business application workload level as well at site level. AWS has multiple availability zones within US and Europe to provide more than one physical location to host the business critical workload instances. Each availability zone is designed with fault separation which means the zones are physically separated within a typical metropolitan region, on different flood plains, in seismically stable areas. In addition to discrete uninterruptable power source and onsite backup generation facilities, they are each fed via different grids from independent utilities to further reduce single point of failure [11]. AWS guarantees "three nines", which means availability $99.9 \%$ of the time. In this way, AWS is considered a "commodity cloud", meaning it fits most of the Non-business Critical (NBC) workloads as long as there are no extenuating enterprise-class requirements, such as high available, stringent SLOs and enterprise-class security. The Unisys Secure Cloud (USC), on the other hand has been audited for most of the compliance processes that are the hallmark of enterprise-class computing, such as support for critical financial business applications or "four nines" availability to customers. Three nines means the computing system can be unavailable up to 8.76 hours a year while four nines translate to a maximum down time of 53 minutes. CDSS helps in considering all the business attributes and assigning related weightings to them for each workload. The inbuilt analytics of CDSS helps in identification of Business Critical (BC) workloads for the organization on the basis of assigned weights for each of the business as well as the technical attributes.

Here are a few of the attributes which uniquely qualify workloads as an enterprise-class workload:

- Workloads composed of multiple, codependent services, and high throughput online transaction processing, e.g., OLTP Applications, Real-time Transaction Processing applications, Online Net-Banking, Airline travel ticket booking applications, Power Grid Management Applications, Public Transport Management Applications

- Health Care Applications with patient data and personal medical information, e.g., Medical insurance, Patient and hospital management systems

- Workloads requiring a high level of regulatory compliance, accountability, e.g., workload subject to Sarbanes-Oxley Federal Government Systems such as Stock Exchange Applications

- Other workloads such as National Defense Systems, Nuclear and Biochemical Laboratory 


\section{Management Applications}

- Any workload which requires $99.99 \%$ uptime or more.

Any workload of a similar nature, which share any of the attributes described above, will be automatically classified as Business Critical workload. Such business critical applications cannot be moved to any commodity public cloud environment due to certain compliance and technology limitations.

Other criteria to identify the workloads that may not be appropriate for the commodity clouds today are -

- Workloads based on 3rd party software which does not have a virtualization or cloud aware licensing strategy

- Workloads requiring detailed chargeback or utilization measures as required for capacity planning or departmental billing

- Workloads requiring a lot of customization and are not written specifically to execute in a web-based environment

- Workloads which depend on sensitive data normally restricted to the enterprise due to security requirements, e.g., employee information, financial information [12].

Table 3 shows the criteria to classify the Business Application (BA) workload as enterprise-class (E) or commodity (C).

Table 3. Workload classification into BC and NBC

\begin{tabular}{|c|c|c|c|c|}
\hline $\begin{array}{c}\text { Business } \\
\text { Workload }\end{array}$ & $\begin{array}{c}\text { Availability } \\
(\%)\end{array}$ & OLTP & $\begin{array}{c}\text { Medical } \\
\text { Applicatio }\end{array}$ & C/E \\
\hline BA1 & 99.9 & No & Yes & E \\
\hline BA2 & 99.0 & No & No & C \\
\hline BA3 & 99.999 & Yes & Yes & E \\
\hline BA4 & 99.9 & No & Yes & E \\
\hline BA5 & 95.0 & No & Yes & E \\
\hline
\end{tabular}

Once a business defines what they consider to be Enterprise or Commodity workloads, the next step is to identify the technical attributes of the workloads under consideration before physically moving them to public cloud.

The technical attributes of the business workloads which CDSS takes into consideration are-

- Size of workload in GBs - If each VM is a $\mathrm{GB}$ in size and you have $1000 \mathrm{VMs}$, it can take over a day to transfer the VMs to the cloud using today's Internet technologies

- Size in GBs of the storage that is required by the application
- Rate at which the backend database changes with every business transaction using the current business application architecture and business logic

- Is conversion required - from physical to virtual $(\mathrm{P} 2 \mathrm{~V})$ or virtual to virtual $(\mathrm{V} 2 \mathrm{~V})$ ?

- Required operating system for the business applications

- Supported database management system

- Frequency of access to storage system

- Level of data encryption required

- Tolerance to individual system or entire site failure

- Dependency on unique hardware and peripherals

- Application licensing

- Ease of installation and configuration in the public cloud environment

- Technical support and expertise required to manage the application

- Frequency of patching and updating the business application

- The volume of database to be synchronized between private data center and cloud environment while moving the workloads

If the business workload is not supported in a virtualized environment, then the workload can be reconfigured only with a physical server machine in the cloud environment. This attribute reduces the choice of cloud providers to host the workload. If the workload is already in virtual image form, such as VMware supported format, then such VMs of the workload can be just moved into a public cloud that supports VMware format. Moving a workload which is in VMware supported format to AWS EC2 cloud environment require $\mathrm{V} 2 \mathrm{~V}$ conversion. AWS business partners like enStratus and CloudSwitch facilitate such V2V conversion for workload deployment. After converting the images from one virtual format to another format, performance and behavior must of entire workload must be tested before physically moving it into the public cloud. This additional level of verification could be avoided by moving the workload to the public cloud provider which supports a similar virtualization format. For example, move VMware workload images to clouds that support VMware images. It is comparatively less risky.

The Table 4 shows further scrutiny of the nonenterprise-class workloads, previously which was identified as commodity (NBC) on the basis of the business attributes of the workloads. 
Table 4. Further filtering of the NBC workload

\begin{tabular}{|c|c|c|c|c|}
\hline $\begin{array}{c}\text { Business } \\
\text { Workload }\end{array}$ & $\begin{array}{c}\text { VM / PM } \\
\text { Hosted }\end{array}$ & $\begin{array}{c}\text { Can be } \\
\text { virtualized }\end{array}$ & $\begin{array}{c}\text { H/W } \\
\text { Dependency }\end{array}$ & $\begin{array}{c}\text { C } \\
\text { R } \\
\text { R }\end{array}$ \\
\hline BA1 & VM & - & No & 1 \\
\hline BA2 & PM & Yes & No & 2 \\
\hline BA3 & PM & No & No & 3 \\
\hline BA4 & VM & - & Yes & 2 \\
\hline BA5 & PM & Yes & Yes & 3 \\
\hline
\end{tabular}

Theworkloadwith thehighestCloudCompatibility Ranking (CCR) will be most preferred to move to the public cloud environment. As ranking goes down, the complexity and risk of moving the workload increases. Few of the workloads in the Table 4 are in VM (virtual machine) form and few are in PM (physical machine) form hosted on the bare metal server hardware.

Hence, the final CCR generated by the CDSS analytics help the organization to identify the most suitable business workloads that can be moved to a public cloud environment without technology or business architecture re-factoring. Figure 3 shows the database in use to manage the workload profile with business and technical attributes of the business service workloads.

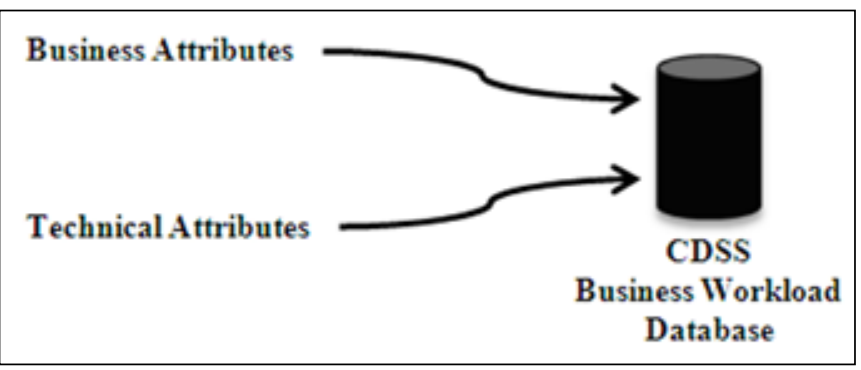

Fig. 3. Business Workload Analysis Database

\subsection{Analytics to Find the Best Match}

So far we have analyzed and classified the public cloud environments on the basis of their offerings, the industry compliance and technical attributes. The CDSS ranks each public cloud environment in Cloud Provider Ranking (CPR) on the basis of how well it aligns with business attributes as well as technical attributes of the workload to be hosted within the environment. The higher the CPR ranking, the more preferred the cloud environment is for a specific type of workload.

Here is an equation for a cloud provider's CPR calculation on the basis of business and technology CPR,

\section{$\mathrm{CPR}_{\text {business }}+\mathrm{CPR}_{\text {technology }}=\mathrm{CPR}_{\text {cloud }}$}

Similarly, the CDSS analyzes the business application workload and classifies it in terms of commodity (NBC) and enterprise-class (BC) workloads. The commodity focused workloads are further analyzed on the basis of its technology attributes. The final analysis gives the workload ranking in CCR. Here, the higher the CCR ranking, the easier is the workload movement to public cloud environment.

\section{Business Workload $\rightarrow$ Commodity (NBC) workload/Enterprise-class (BC) workloads $\rightarrow$ CCR $_{\text {TECHNOLOGY }} \rightarrow$ CCR WoRKLOAD}

CDSS uses the best combination of the CPRCLOUD of the public cloud providers and the CCRWORKLOAD of the business service workload to find the best-fit for the business workload.

\section{CONCLUSION}

The benefits and risks of moving to cloud computing are not easy to assess. Detailed work needs to be done to ensure that the total cost of ownership (TCO), security, confidentiality, functionality, integration and process consistency requirements are considered [9].

As an organization considers the use of cloud computing, and then focuses on picking specific clouds for their workloads, a CDSS helps guide the discovery of information as well as suggesting specific implementations. This reduces the requirement to invest time and money to hire third party workload and public cloud assessment service providers to accomplish the analysis. Once the CDSS exercise is complete, it can be used quite easily to re-analyze as information changes - either regarding the workload attributes or the cloud attributes.

\section{REFERENCES}

[1] Gartner EXP Worldwide Survey of Nearly 1,600 CIOs Shows IT Budgets in 2010 to be at 2005 Levels, Gartner Inc. Stamford, 2010 http://tinyurl.com/GartnerEXP

[2] Johnson S. 2009. Cloud Computing Types: Public Cloud, Hybrid Cloud, Private Cloud. CircleID (Iomemo Inc.) (Mar 2009). http:// tinyurl.com/CCTypes

[3] Aberdeen Insights - Strategy, Figure 8, Cloud User IT Cost Savings. Aberdeen Group, (Sep 2009)

[4] Booz Allen Hamilton. The Economics of Cloud Computing. http:// tinyurl.com/BoozAllenH

[5] Martin B. 2008. Twenty Experts Define Cloud Computing. SYS-CON Media Inc. http://tinyurl.com/SCMedia

[6] Data Center Site Infrastructure Tier Standard: Topology, 1-4. Uptime Institute, LLC. 2009. http://tinyurl.com/UpTimeInstitute

[7] Rimal B. P., Choi E., Lumb I. 2009. A Taxonomy and Survey of Cloud Computing Systems. Fifth International Joint Conference on INC, IMS and IDC, (Aug 2009), 45. http://tinyurl.com/CloudTaxonomy

[8] The Open Virtual Machine Format Whitepaper for OVF Specification, 7. Version 0.9. VMware, Inc and XenSource, Inc. http://tinyurl.com/ VXOVF

[9] Drobik A., Woods J., Collins K., Holincheck J., Maoz M., White A., Desisto R. P. 2009. Business Applications and Cloud Computing: Convergence or Myth?. ID Number: G00168840, 1 (July 2009), Gartner, Inc. http://tinyurl.com/BusinessACC

[10] Kwang K. 2009. Assess business needs first before cloud move. ZDNet Asia, (Nov 2009). http://tinyurl.com/zdnetasiaN2009 
[11] Amazon Web Services: Overview of Security Processes 2009. Amazon. com, Inc. (Nov 2009), 7-8. http://tinyurl.com/AWSSWP

[12] Ott B. Moving Applications to the Cloud - Determining What Applications Make Sense for Your Business. Webinar. 2009. Unisys Corporation. 20-21. http://tinyurl.com/UnisysWebinar

[13] Salsburg M. A, Ph.D. The Perfect Storm for Enterprise-Class Clouds. 2009. Unisys Corporation. (June 2009), 10 http://tinyurl.com/ UnisysECC

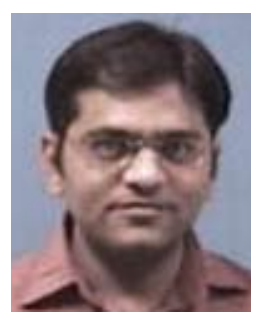

Mohammad Firoj Mithani

Mohammad Firoj has over 10 years of work experience in IT infrastructure management, support and consulting. He is a Master of Computer Applications (MCA) and a Project Management Professional (PMP). He has over 12 vendor certifications including VCP, CPSA, ITIL and MCTS. He has been awarded one international patent for business workload and cloud assessment analytics. His research interest is in the area of cloud computing and IT infrastructure virtualization.

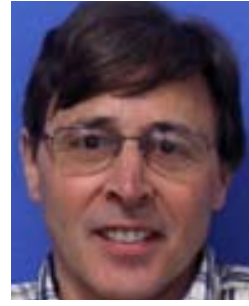

Michael A. Salsburg, Ph. D.

Dr. Salsburg has published and presented over 60 papers internationally in the fields of infrastructure and business service modeling, management and optimization. His current focus within Unisys is the realization of real-time infrastructure management. He has been awarded three international patents, out of which two are for optimization and modeling algorithms. He received his Ph.D. in Mathematics from Drexel University in 1992 in the field of Probability and Statistics.

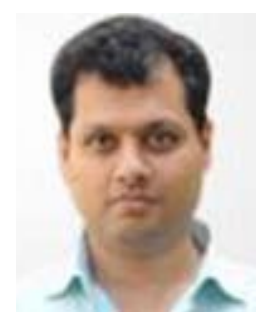

Shrisha Rao, Ph. D.

Dr. Rao, a faculty at IIIT-Bangalore, received his $\mathrm{Ph} . \mathrm{D}$. in computer science from the University of Iowa, and also has an M.S. in logic and computation from Carnegie Mellon University. His primary research interest is in the area of distributed computing, specifically algorithms and formal methods for concurrent and distributed systems. He also has interests in problems such as distributed fair division and demand-side management. $\mathrm{He}$ occasionally dabbles in mathematics. 